\title{
Transient characteristics of BCB encapsulated surface micromachined Aluminum micro-mechanical resonators
}

\author{
Sayanu Pamidighantam \\ Indian Institute of Science (IISC) \\ Supercomputer Education and Research Center (SERC), IISC, Bangalore 560012, India. \\ Email:sghantam@serc.iisc.ernet.in, Phone: 009180 23600654, extn:314.
}

\begin{abstract}
In this contribution, the transient characteristics of Benzo Cyclo Butene (BCB) encapsulated Aluminum (Al) micro mechanical resonators as a function of time varying pressure are studied. The quality factor of encapsulated $\mathrm{Al}$ resonator showed a drastic change with time of more than $200 \%$ reduction. The estimated transient leak rate spans over several orders of magnitude $\left(10^{-5}\right.$ to $\left.10^{-9} \mathrm{~atm} \mathrm{~cm}^{3} \mathrm{~s}^{-1}\right)$. A theoretical description of $\mathrm{Al}$ micro mechanical resonator and the leak rate are presented. Possible causes for the leak rate and the potential solutions are also outlined.
\end{abstract}

\section{Introduction}

It is obligatory for all the electronic components to operate in a packaged environment. In technical terms, the controlled packaged environment is called hermeticity. The extent of hermeticity however varies for different applications. The severity of hermeticity specification increases from commercial/mass market products to military/defense and space products in that order. For these reasons, the study of the packaged components and the hermeticity in particular forms an important device characterization study. Among the many techniques developed to characterize various packaging specifications, leak testing of sealed electronic components not only provides a measure of hermetic nature of the seal but also dictates the overall cost of the product.

The standard measurement technique used for leak testing is known as back pressuring method. Simply defined the technique of leak testing involves a two step process. Firstly, the device is pressurized in a tracer gas during which the gas enters the object through any possible leak source. The second step involves the detection of re emission of the tracer gas. The rate of emission depends not only on the leak size, but also on how much gas has entered during the pressurizing perid. The practical implementation involves a third stage in addition to the two steps described - the transit stage. It was pointed out that transit stage can become a significant bottleneck in accurate leak rate measurement [1]. This is especially true in the case of small cavity sizes occupied by MEMS devices. Typically the cavity volumes of MEMS devices fall in the range of $10^{-5} \mathrm{Cm}^{3}$ to $10^{-2} \mathrm{Cm}^{3}$. The smallness of the MEMS cavity sizes, the suspended beam structures that form an integral part of the MEMS device, the hermeticity requirement, the requirement for stable and reliable functioning of MEMS device and the associated cost together makes packaging of MEMS a prominent area of research. Among the different classes of MEMS devices, packaging technologies required for RF-
MEMS devices warrants a low thermal budget process. The requirement that RF-MEMS devices should be operated in a controlled ambient is well studied by S. T. Patton and J. S. Zabinski [2]. They found a significant reduction in the actuator life time under high vacuum and high relative humid conditions suggesting that an optimum vacuum and relative humidity $(\mathrm{RH})$ condition are needed to operate these devices.

Low thermal budget BCB encapsulated RF-MEMS switches were demonstrated by Jourdain et. al [3]. Despite the excellent properties of $\mathrm{BCB}$ material, the question remains about the hermetic nature of this material. Further it was pointed out in reference [3] that in spite of the excellent gross leak characteristics of BCB material, the fine leak characteristics of micro cavities is a matter of concern to the MEMS researchers. Therefore it is essential to study and understand the response behavior of $\mathrm{BCB}$ material to fine leak to resolve the outstanding issues concerning the fine leak characteristics.

In this contribution, the transient characteristics of $\mathrm{BCB}$ encapsulated Aluminum (Al) micro mechanical resonators are studied. Theoretical presentaton on micromechanical resonator, quality factor and their connection to the leak rate is given in Section. 2, experimental set-up is described in Section. 3, measurement results are presented in Section. 4, followed by discussions and conclusions in Section. 5 and references in Section. 6 respectively.

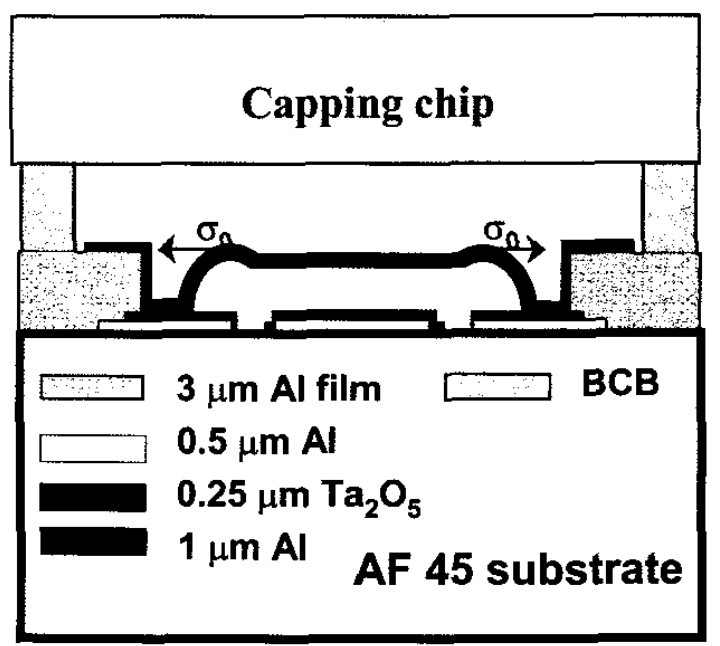

Fig. 1. Schematic representation of $\mathrm{BCB}$ encapsulated $\mathrm{Al}$ micro resonator (Figure not to scale) 


\section{Theoretical Analysis}

The schematic picture of a micro-mechanical fixed-fixed $\mathrm{Al}$ beam structure ( $\mathrm{CC}$ beam) having bi-axial stress and the process induced step-up beams is shown in Fig. 1. For such a beam structure, it can be shown using approximate methods that the angular resonance frequency is equal to [46]

$$
\begin{aligned}
& \omega=\omega_{0} \sqrt{1+\frac{3 \mu \mathrm{X}_{\mathrm{AC}}^{2}}{4 \omega_{0}^{2}}} \\
& \omega_{0}=6.46 \sqrt{\frac{\frac{\hat{\mathrm{E} b h}{ }^{3}}{\mathrm{le}^{4}}+\frac{054 \hat{\sigma}_{0} \mathrm{bh}}{1 \mathrm{e}^{2}}-\frac{0.024 \mathrm{bV}{ }^{2} \varepsilon_{0}}{(1-\beta)^{3} \mathrm{~d}_{0}^{3}}+\mathrm{K}_{\mathrm{a}}}{\rho \mathrm{A}}} \\
& \mathrm{K}_{\mathrm{a}}=\frac{0.024 \mathrm{~Pb}}{(1-\beta) \mathrm{d}_{0}}
\end{aligned}
$$

The effects due to soft spring and the hard spring mechanisms are taken into account in eqn. (1). Further $\mathrm{X}_{\mathrm{AC}}$ is the amplitude of the vibration, $\hat{E}$ is the effective elastic modulus, $\hat{\sigma}_{0}$ is the residual bi-axial stress of structural $\mathrm{Al}$ film, $\mu$ is the parameter that dictates the hard spring or soft contribution for free vibrations of beam, le, b, h, and $d_{0}$ are the dimensional parameters, viz, length, width, thickness and the gap-spacing, $\rho$ is the $\mathrm{Al}$ film material density, $\mathrm{P}$ is the ambient pressure at which the resonator is operating, $\varepsilon_{0}$ is the permittivity of free space, $\mathrm{V}$ is the applied DC bias and $\beta$ is the ratio of maximum displacement due to the applied DC bias to the original undisturbed gap-spacing respectively. The angular resonance frequency of $\mathrm{Al}$ microresonator is denoted by $\omega_{0}$, where as the forced vibrations due to the external excitation force by $\omega$ respectively. The damping force has two contributions, one proportional to the velocity of the beam, and the other proportional to the acceleration [7]. The acceleration component of damping force further introduces a term in the denominator of eqn. (1) (but not considered here). In general, there are various mechanisms that alter the resonance frequency. The axial force increases the resonance frequency, the application of DC bias reduces beam bending stiffness, the damping component that is proportional to the acceleration increases the effective mass, and a large squeeze number enhances the beam stiffness respectively.

Of particular importance to the current analysis is the quality factor of the Al micro-mechanical beam resonator. The quality factor is defined as the ratio of the stored vibrational energy to the dissipated energy over one time period. The quality factor of a micro resonator is a function of many variables such as ambient pressure, the type of gaseous species, ambient temperature, intrinsic material losses such as thermo elastic frictional forces [8], anharmonic effects [9], surface related effects, the type of dopants present in the structure [10], the finite non-zero absorbance of elastic energy into the fixed support regions. The overall effective loss is sum of all the losses. Consequently, the effective Q-factor is sum of inverses of the individual contributing Q-factors.
Fortunately majority of these loss mechanisms show a frequency dependence. Thus if a micro resonator is designed for a specific resonance mode and frequency, some of the loss mechanisms can be made negligible. For instance, thermo elastic frictional forces exhibit a characteristic frequency and if a micro resonator is designed far away from this frequency, the loss incurred due to the thermo elastic frictional forces can be safely ignored. On a similar note, the energy loss into support spring is found to be dominant when the dimension of the micro resonator is of the same order as that of the wave length of acoustic wave. Consequently, for a structure of dimension that is several times greater than that of the acoustic wave length, the effects due to elastic supports can be eliminated from the analysis.

Three prominent regions exist for charcterizing the Qfactor, viz, intrinsic region $\left(Q_{\text {int }}\right)$, molecular region $\left(Q_{\text {mol }}\right)$, and the viscous damping region $\left(\mathrm{Q}_{\mathrm{vis}}\right)$. Based on the basic definition of quality factor, the following expressions for the Q-factor can be derived for each of these three regions [7, 11]. The basic expression for the quality factor is given by

$$
Q=\frac{\omega\left(\rho l e b h+\frac{\beta_{2}}{\omega}\right)}{\beta_{1}}
$$

Where $\beta_{1}$ and $\beta_{2}$ are the coefficients of damping force corresponding to the velocity component and the acceleration component acting upon the vibrating body [7]. The $Q$-factor in the molecular region $\left(Q_{\text {mol }}\right)$ and in the viscous region $\left(Q_{\mathrm{vis}}\right)$ are given by $[4,10]$

$$
\begin{aligned}
& Q_{\text {mol }}=\frac{31.57 \omega \rho l e b h}{P^{2}} \\
& Q_{\text {vis }}=\frac{\omega \rho l e b h+3 \pi R^{2} \sqrt{\frac{\left.2 \eta_{\text {eff }}(P)\right)_{0} P M}{R_{0} T}}\left(1+\frac{2 R}{9 \delta}\right)}{6 \pi \eta_{\text {eff }}(P) R\left(1+\frac{R}{\delta}\right)} \\
& \delta=\sqrt{\frac{2 \eta_{\text {eff }}(P) R_{0} T}{\omega P M}} ; P=P 0\left(1-e^{\left.\frac{-L t}{P_{0} V_{0}}\right)}\right.
\end{aligned}
$$

where and $\eta_{\text {eff }}$ is the effective viscosity of the medium, $R_{0}$ is the gas constant, $R$ is the characteristic dimension, $M$ is the the air mass, $\mathrm{T}$ is the temperature, $\mathrm{V}_{0}$ is the volume of the cavity, $\mathrm{L}$ is the leak rate in the units of $\mathrm{Atm}_{\mathrm{Cm}} \mathrm{s}^{-1}$, and $t$ is the duration over which the cavity is subjected to the external pressure, $\mathrm{P}_{0}$.

An important design parameter is the transition pressure between the viscous regime and the molecular regime. The transition pressure defined as the pressure at which the $Q_{m o l}$ and $\mathrm{Q}_{\mathrm{vis}}$ are equal. The fact that micro resonator's characteristics are sensitive to pressure variation becomes evident upon inspecting eqn. (3). This is precisely the reason for selecting $\mathrm{Al}$ micro resonator as test vehicle to study the leak rate aspects of $\mathrm{BCB}$ material. In order to estimate the leak rate (L), it is important to link the Q-factor to the leak rate and this is done by expressing the pressure variation in the cavity to the leak rate. 


\section{Experimental Procedure}

The Al micro resonators are fabricated by employing a novel surface micromachining process described in [12]. After the release of the structures, the resonator structures are encapsulated using BCB (Cyclotene sereis 4000) as the bonding layer making use of the procedure illustrated in Fig. 2. It consists of two wafers, one device wafer wherein the Al micro-mechanical resonators are fabricated and another wafer consisting of patterned $\mathrm{BCB}$ layer and diced into individual chips used as capping wafer. The volume of the cavity formed after bonding and determined by the BCB layer thickness and the lateral area of MEMS device is approximately equal to 90 nano litres $\left(10^{-4} \mathrm{Cm}^{3}\right)$. For the dimension of $\mathrm{le} \sim 400 \mu \mathrm{m}, \mathrm{b} \sim 17 \mu \mathrm{m}, \mathrm{h} \sim 1 \mu \mathrm{m}, \mathrm{d}_{0} \sim 2.5 \mu \mathrm{m}$, and density of $\rho \sim 2300 \mathrm{kgm}^{-3}$, the estimated tranistion pressure, $P_{\text {trans }}$ is found to be in the range of $4 \mathrm{mB}$ to 0.76 $\mathrm{mB}$ for characteristics lengths ranging from $10 \%$ of beam length to full beam length.
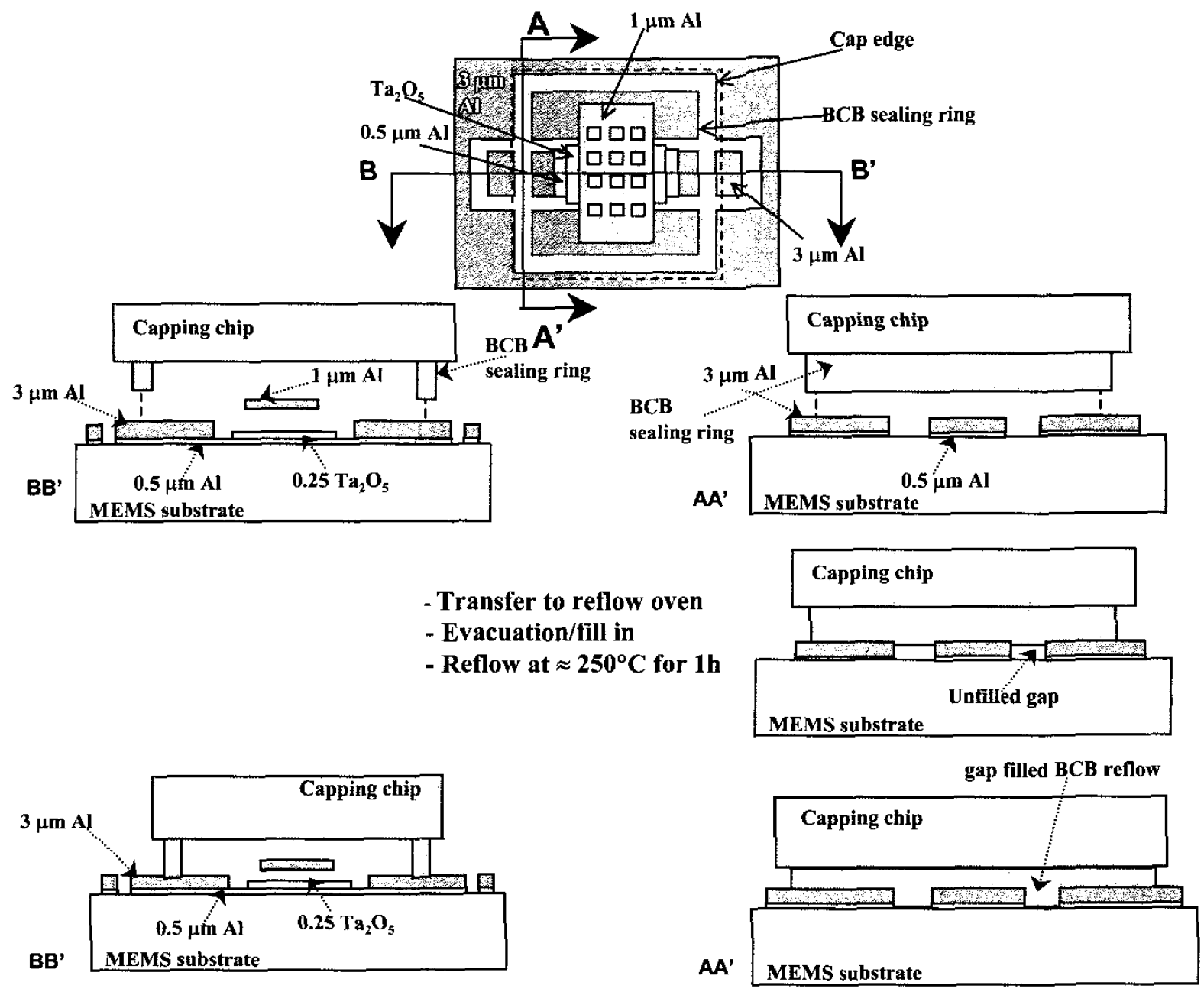

Fig. 2. The procedure of chip on wafer encapsulation scheme (Figure approximately to scale) 


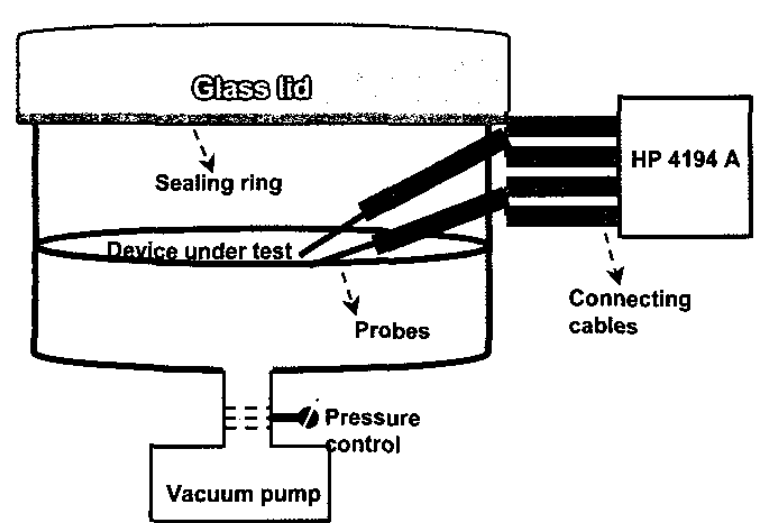

Fig. 3. The schematic of vacuum measurement set-up

The schematic representation of the measurement set up is shown in Fig. 3. The experimental set up shown in Fig. 3 is equipped with electrical cables entering into the chamber through the openings that are leak tight. A rough mechanical pump is employed to evacuate the chamber pressure. In order to study the response behavior of the $\mathrm{BCB}$ layer to the chamber pressure, that is how soon the cavity pressure is equalized to the chamber pressure, the pressure is increased in a controlled fashion after reaching the lowest pressure with the help of pressure knob shown in Fig. 3. During this stage at each pressure point the impedance/conductance of excited $\mathrm{Al}$ micro resonator has been recorded as a function of time. After reaching the atmospheric pressure, the microresonator's response is measured again as a function of time.

\section{Measurement Results}

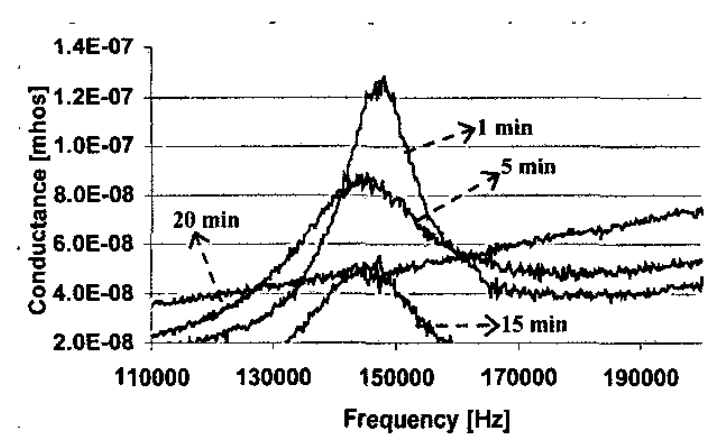

Fig. 4. The measured transient resonance characteristics after reaching 1 Bar pressure (reached from vacuum)

The plot describing the transient characteristics of $\mathrm{Al}$ micro-resonator at atmospheric pressure is shown in Fig. 4. It is clear from Fig. 4 that the response of $\mathrm{Al}$ microresonator measured immediately after reaching atmospheric pressure exhibits a sharper and as a result higher quality factor.

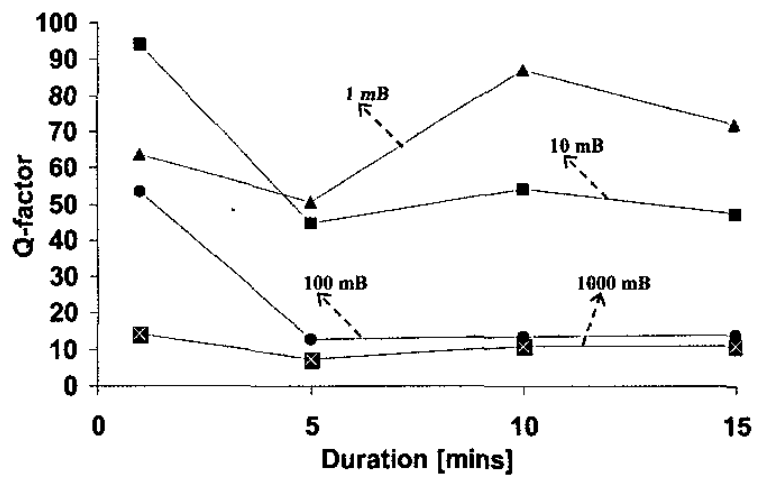

Fig. 5. Extracted Q-factor as function of time at various pressure conditions

The quality factor is extracted by estimating the bandwidth corresponding to 0.7 times the maximum amplitude and by taking the ratio of center frequency to the bandwidth (This corresponds to Q-factor at $3 \mathrm{~dB}$ bandwidth). By and large the variation in Q-factor shows a predictable behavior as a function of time as shown in Fig. 5 . However, in the curve corresponding to $1 \mathrm{mB}$ pressure exhibits rise in Q-factor at 10 minutes and 15 minutes duration. This can be interpreted as follows. The resonance spectrum corresponding to $1 \mathrm{~min}$ and $5 \mathrm{~min}$ intervals are sharper and could not be properly captured from the measured data. This fact was also evident from the measured data-the amplitude of conductance coresponding to $1 \mathrm{~min}$ is smaller than that of 5,10 and 15 minutes plot respectively (not included in the manuscript).

A more refined sampling in a narrow frequency range is required to capture a sharp resonance. Sometimes the resonance is so sharp it becomes difficult to extract the quality factor without increasing the number of measurement points. This is especially true in the case of high vacuum measurement. As time progresses, the pressure inside the cavity and outside the cavity are equalized and the air molecules enter through the finite openings of BCB layer eventually reaching an equilibrium state. There are many possibe sources for the existence of fine leak. For instance it might be possible that the reflow process conditions of BCB are not optimum to facilitate gap filling (shown in Fig. 2). Another possible explanation is the presence of structural imperfections created in the $\mathrm{BCB}$ layer due to stress buildup or during the bonding cycle. These structural imperfections could in turn create openings for air passge. 


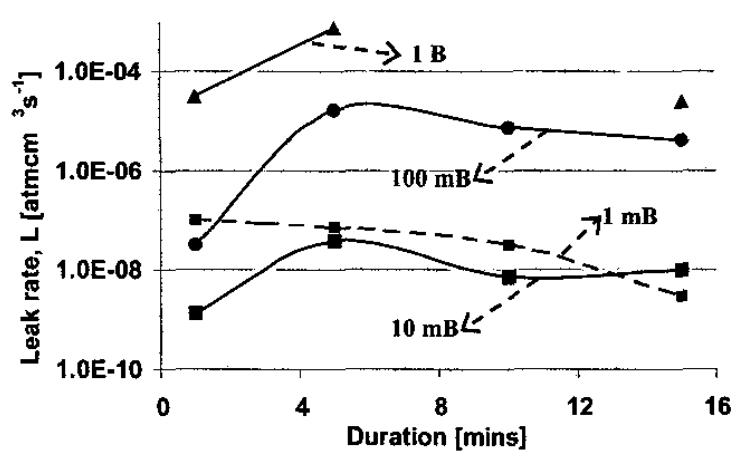

Fig. 6. Estimated leak rate during transient stage

The fact that the transition pressure is in the range of $\mathrm{mB}$ means that quality factor corresponding to viscous regime should be used to estimate the leak rate (majority of experimental pressure conditions are above $1 \mathrm{mB}$ ). The extracted leak rate during the transient stage has been captured and shown in Fig. 6 . The leak rate is estimated by making use of eqn. (3). In using eqn. (3), in addition to the dimensional values mentioned, the following numerical values are assumed.

$$
\mathrm{R}_{0}=8320 \mathrm{Kgm}^{2} \mathrm{~s}^{-2} \mathrm{~K}^{-1} ; \mathrm{M}=29 \mathrm{Kg} ; \mathrm{T}=300 \mathrm{~K} ;
$$

The pressure dependence of effective viscosity mentioned in reference [13] are taken into account in estimating. The value of $\eta_{\text {eff }}$ varies between $8.5^{*} 10^{-6}$ to $1.8^{*} 10^{-5}\left(\mathrm{Nsm}^{-2}\right)$ in the pressure range of $1 \mathrm{mB}$ to 1 Bar.

The complete analysis on leak rate behavior captured in Fig. 6 is beyond the scope of this manuscript and requires further investigation. However, several points are worth mentioning refering to Fig. 4, Fig. 5 and Fig. 6 . It is interesting to note from Fig. 6, the evidence of vulnerability of small cavity sizes of MEMS structures to the existence of fine leak.

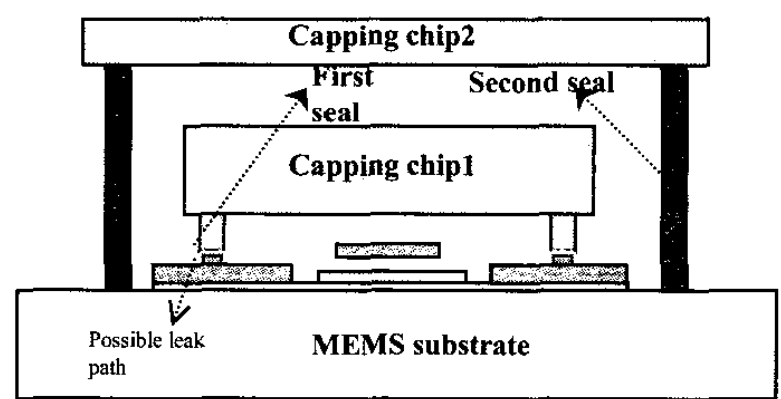

Fig. 7. A potential two level solution to minimize fine leak in MEMS cavities

It is rather more important to discuss the potential solutions to minimize or eliminate the fine leak in ultra small MEMS cavities. One possible solution is presented in
Fig. 7. Here a second sealing covers over the already existing encapsulation. By introducing a second seal, the effective cavity volume is increased. The increased volume improves the fine leak performance. However, this approch consumes more chip area. The fine leak can also be minimized by exploring alternative flowable polymer materials with improved planarization capability or by minimizing the topography variation on device wafer.

\section{Conclusions}

The main emphasis given in this contribution is the development of a characterization procedure for detecting fine leak characteristics of MEMS cavities. The performance of $\mathrm{Al}$ micro-mechanical resonator is found to be sensitive to ambient pressure inspite of being encapsulated with BCB. It was successfully demonstrated that the in-situ vacuum characterization of encapsulated Al micro-mechanical resonators is an ideal tool for detecting fine leak. With the methodology and the technique presented in this contribution, it is possible to detect leak rates of the order of $10^{-10} \mathrm{~atm} \mathrm{~cm}^{3} \mathrm{~s}^{-1}$ during transisent stage. In addition, the described in-situ characterization provides insight into the physical mechanisms that take place and helps in understanding the device physics. Possible causes for the leak rate and the potential solutions are discussed. Further it was elucidated that the solution to leak tightness lies in techology, materials development as well as novel test vehicles that can capture the true nature of hermeticity of a micro cavity.

\section{Acknowledgments}

The author would like to acknowledge the help provided by Prof. N. Balakrishnan and Prof. S. M. Rao of SERC, IISC as invaluable and unforgettable. This work was part of the doctoral work carried out at MEMS lab, IMEC, Belgium.

\section{References}

1. Howl. D. A. et al, "The back-pressuring technique of leak testing", Vacuum, Vol. 15, No. 7, 1965, pp. 347 352.

2. Patton. S. T. et al, "Failure mechanisms of a MEMS actuator in very high vacuum", Tribology International, Vol. 35, No. 6, 2002, pp. 373-379.

3. Jourdain. A. et al, "Investigations of the hermeticity of BCB-sealed cavities for housing (RF-)MEMS devices", Proceedings of the $15^{\text {th }}$ Annual International Conference on MicroElectroMechanicalSystems (MEMS), Las Vegas, Jan, 2002, pp. 677.

4. Thomson.W. T. et al, Theory of vibration with Applications, $5^{\text {th }}$ edition, Pearson Education, (Singapore, 1998).

5. Andrews. M. et al, "A comparison of squeeze-film theory with measurements on a micro structure", Sensors and Actuators A, Vol. 36, 1993, pp. 79-87.

6. Kokubun. K. et al, "Unified formula describing the impedance dependence of a quartz oscillator on gas 
pressure", Journal of Vacuum Science Technology, A, Vol. 5, No. 4, 1987, pp. 2450-2453.

7. Landau. L. D. et al, Fluid Mechanics, Course of Theoretical Physics, Vol. 6, Pergamon, (Oxford, 1965).

8. Roszhart. T. V, "The effect of thermoelastic internal friction on the Q of micromachined silicon resonators", Technical Digest of the IEEE Solid-State Sensors and Actuator Workshop, Salt Lake City, Utah, Feb. 1989, pp. 53-59.

9. Buser. R. A. et al, "Resonant Silicon Structures", Sensors and Actuators, Vol. 17, No. 1/2, pp. 145-153.

10. Nguyen. C. T. - C, "Frequency-Selective MEMS for miniaturized communication devices", Proceedings of IEEE Aerospace Conference, Colorado, March 21-28, 1998, Vol. 1, pp. 445-460.

11. Blom. F. R. "Dependence of the quality factor of micromachined silicon beam resonators on pressure and geometry", Journal of Vacuum Science and Technology B, Vol. 10, No. 1, 1992, pp. 19-26.

12. Naga Sayanu Pamidighantam, Doctoral dissertation, ISBN number: 90-5682-486-4, March 2004.

13. Andrews. M. K., "A resonant pressure sensor based on a squeezed film of gas", Sensors and Actuators, A, Vol. 36,1993 , pp. 219-226. 